\title{
Comparison of regularizations of vortex sheet motion
}

\author{
Monika Nitsche ${ }^{\mathrm{a}, *}$, Mark A. Taylor ${ }^{\mathrm{b}}$, Robert Krasny $^{\mathrm{c}}$ \\ ${ }^{a}$ University of New Mexico, Department of Mathematics and Statistics, Albuquerque, NM 87131, USA \\ ${ }^{b}$ Los Alamos National Laboratory, Computer and Computational Sciences Division, Los Alamos, NM 87545, USA \\ ${ }^{c}$ University of Michigan, Department of Mathematics, Ann Arbor, MI 48109, USA
}

\begin{abstract}
This paper presents a numerical comparison of two regularizations of the Euler equations, namely, the vortex blob regularization and regularization by physical viscosity. The initial condition is a flat vortex sheet whose vorticity has been smoothed by convolution. The sheet rolls up into a vortex pair. We compute the frequency of oscillations in the core vorticity and scale it using results for a semi-infinite vortex sheet. The computations indicate that as the smoothing parameter $\delta$ and the viscosity $v$ decrease, the scaled frequency in the vortex blob solutions differs from the one in the Navier-Stokes solutions.
\end{abstract}

Keywords: Vortex sheets; Vortex blob method; Regularization of Euler equations

\section{Introduction}

The vortex sheet is a singular model for a shear layer in which the layer is assumed to have zero thickness. Even with analytic initial conditions, vortex sheets develop singularities in finite time (for a review, see [1-3]). The approach to computing the sheet's motion past the critical time is to regularize the flow and define the vortex sheet as the solution in the limit of zero regularization. Different regularizations are possible. The vortex blob method convolves the singular kernel in the Biot-Savart law with a smooth function. Here we use a regularization which introduces a smoothing parameter $\delta$ into the denominator of the integrand [4-7]. This is numerically convenient, but the true physical regularization is by viscosity. In $2 \mathrm{D}$, it is known that for both the vortex blob and the viscous regularization, the limit of zero regularization yields a weak solution to the Euler equations, provided the initial vorticity is of one sign [8-10]. However, it is not known whether this limit is unique or whether it depends on the regularization.

In [11], we computed the roll-up of an initially flat vortex sheet into a vortex pair and observed irregular features in the vortex core (see Fig. 1a). These features are caused by the onset of chaos in a resonance band, which in turn is caused by oscillations in the core vorticity. The oscillations are not due to external forcing but are self-induced and

* Corresponding author. Tel.: +1 (505) 277-5039; Fax: +1 (505)

277-5505; E-mail: nitsche@math.unm.edu are attributed to a self-induced strain field. These results were unexpected since they were not predicted by similarity theory for semi-infinite vortex sheets [12] and were also not seen in previous vortex blob computations [6]. The question therefore arises whether the irregular features are an artifact of the particular regularization, or whether they occur as well with regularization by viscosity, for small enough viscosity. In the latter case one would expect the irregular features to be present in real fluid flows.

This question led to the present comparison study between vortex blob and Navier-Stokes computations. For the Navier-Stokes computations, the initial vortex sheet is regularized by the parameter $\delta$. Following [13], we study solutions to the vortex blob equations and to the NavierStokes equations with constant viscosity $v$, in the limit in which both $\delta$ and $v$ vanish. The results in [13] give evidence that in this limit, large scale features such as the outer spiral turns at a fixed time $t>0$ converge to the same limit for the two regularizations. Similar agreement was observed between computation and experiment [14]. We observe the same trends in the present computations, but we have not seen any indication yet that the viscous solution develops the irregular features present in the vortex blob solution (Fig. 1b). To gain some insight, here we investigate the oscillations in the core vorticity. It turns out that the oscillation frequency is unbounded as the smoothing parameter vanishes. In order to better compare the frequency in this limit it is useful to rescale it using results for a semi-infinite vortex sheet, described next. 

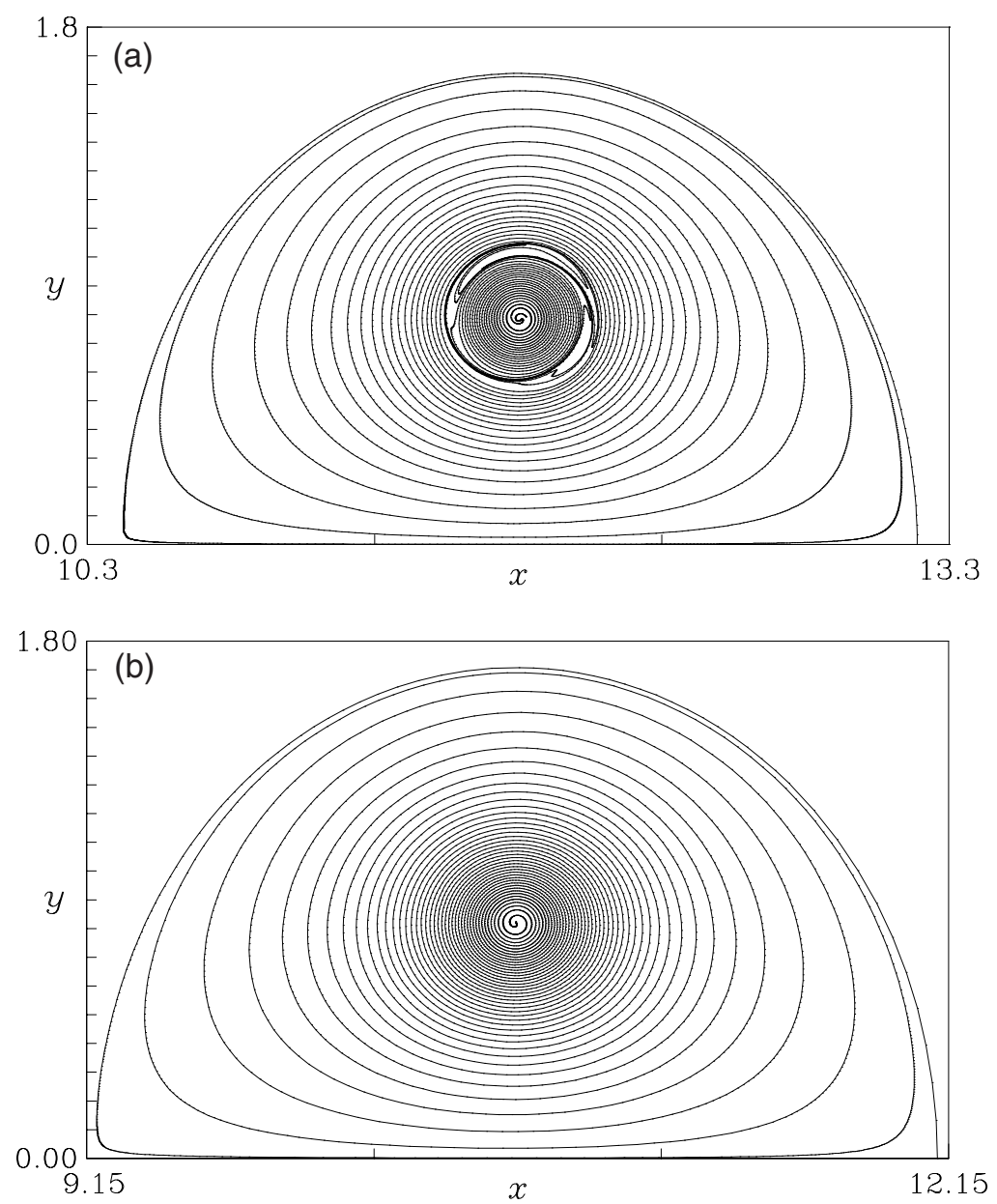

Fig. 1. Position of half the rolled-up vortex sheet at $t=120$. (a) Vortex blob computation, $\delta=0.2$ [11], (b) viscous computation, $\delta=0.2$, $v=10^{-7}$.

\section{Semi-infinite vortex sheet}

The evolution of a regularized semi-infinite vortex sheet is described by the solution $(x(\Gamma, t), y(\Gamma, t))$ to the equations

$$
\begin{gathered}
\frac{\mathrm{d} x}{\mathrm{~d} t}=\frac{1}{2 \pi} \int_{0}^{\infty} \frac{y-\tilde{y}}{(x-\tilde{x})^{2}+(y-\tilde{y})^{2}+\delta^{2}} \mathrm{~d} \widetilde{\Gamma}, \\
x(\widetilde{\Gamma}, 0)=0, \\
\frac{\mathrm{d} y}{\mathrm{~d} t}=\frac{-1}{2 \pi} \int_{0}^{\infty} \frac{x-\tilde{x}}{(x-\tilde{x})^{2}+(y-\tilde{y})^{2}+\delta^{2}} \mathrm{~d} \widetilde{\Gamma}, \\
y(\Gamma, 0)=\frac{-\Gamma^{2}}{2}, \quad \Gamma \in(0, \infty) .
\end{gathered}
$$

The true evolution satisfies Eq. (1) with $\delta=0$. In that case, there is no length scale present and the solution is self-similar. In the vortex blob method $\delta$ is set to a positive value. The solution to Eq. (1) is then no longer self-similar, since $\delta$ introduces a length scale. However, for any constant $T$, Eq. (1) remains invariant under the scaling

$\widehat{x}=\frac{x}{T^{2 / 3}}, \widehat{y}=\frac{y}{T^{2 / 3}}, \widehat{\Gamma}=\frac{\Gamma}{T^{1 / 3}}, \widehat{t}=\frac{t}{T}$ and $\widehat{\delta}=\frac{\delta}{T^{2 / 3}}$.

This implies that the solution computed with a given value of $\delta$ at a given time $t$ is exactly the same, up to scaling, as the solution computed with $\widehat{\delta}$ at time $\widehat{t}$. Equivalently, two solutions are identical up to scaling if

$\frac{\widehat{t}}{t}=\left(\frac{\widehat{\delta}}{\delta}\right)^{3 / 2}$,

as can be seen by eliminating $T$ from the last two equations in Eq. (2). Computations show that the roll-up of a regularized semi-infinite vortex sheet given by Eq. (1) has oscillations in the core vorticity and irregular features. Since the oscillation frequency $f$ scales as the inverse of 
time, it follows from Eq. (3) that

$f \delta^{3 / 2}=\widehat{f} \widehat{\delta}^{3 / 2}$.

A given value of $\widehat{\delta}$ leads to an oscillation frequency $\widehat{f}$. Any other value of $\delta$ will lead to an oscillation frequency $f$ that is related to $\widehat{\delta}$ and $\widehat{f}$ by Eq. (4).

\section{Numerical comparison}

We consider the vortex sheet induced by an impulsively started flat plate [11]. For the Navier-Stokes equations, the initial vorticity is regularized by the parameter $\delta$ and then evolved using viscosity $v$. For each $\delta$, we therefore obtain a vortex blob solution and a sequence of viscous solutions with various values of $v$. We investigate the limit in which both $v$ and $\delta$ vanish. The Navier-Stokes equations are solved using a 4 th order finite difference scheme whose details will be given elsewhere.

The results in [11] show that after an initial transient, the vortex sheet has rolled up into a vortex pair. The vorticity contours in the core are elliptical in shape and the ellipticity oscillates almost periodically in time. Fig. 2 records the oscillation frequency for the vorticity contour $\omega=\omega_{\max } / 2$, where $\omega_{\max }$ is the maximum vorticity, for decreasing values of $\delta$. In view of Eq. (4), we plot the scaled oscillation frequency $f \delta^{3 / 2}$. The figure shows that $f \delta^{3 / 2}$ is approximately constant. Note that based on the

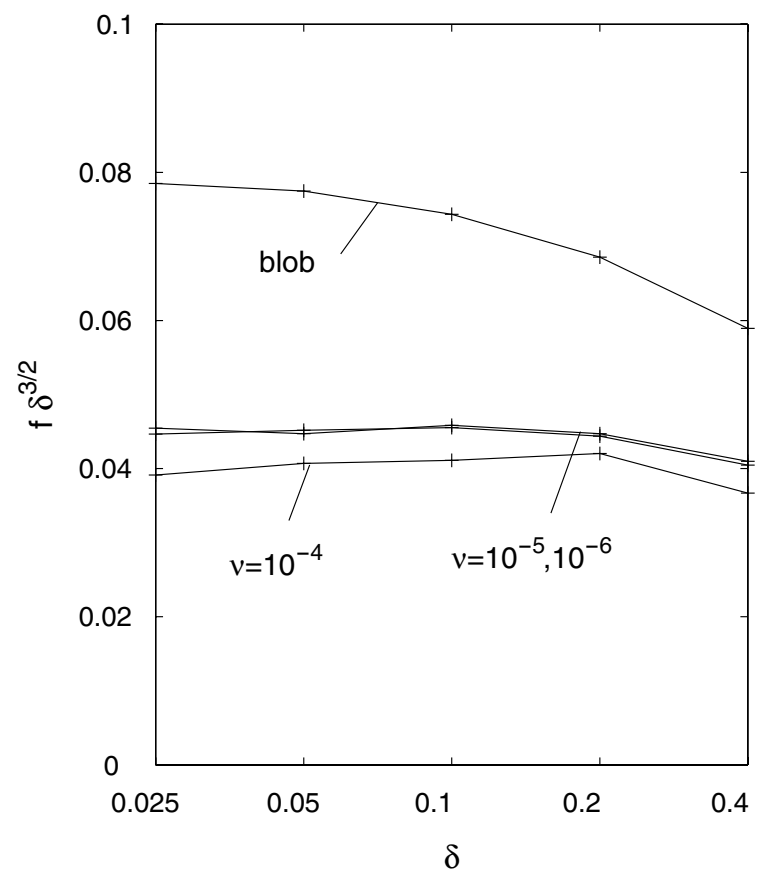

Fig. 2. Scaled oscillation frequency $f \delta^{3 / 2}$ vs. $\delta$ for the vortex blob. discussion in Section 2, $f \delta^{3 / 2}$ is identically constant for a semi-infinite vortex sheet; the fact that the values in Fig. 2 vary slightly with $\delta$ is attributed to the fact that we are dealing here with a finite vortex sheet.

Fig. 2 also shows that as $\delta$ decreases, the viscous values of $f \delta^{3 / 2}$ differ by approximately a factor of two from the vortex blob values, even as $v \rightarrow 0$. We also found (not shown here) that the blob solution is qualitatively different in that all contour levels oscillate at the same frequency, whereas in the viscous case the frequency increases towards the center of the core. In the workshop we will document these and other observations. We note that the current results about the scaled oscillation frequency do not address the question of whether the irregular features in the vortex blob solution also occur in the Navier-Stokes solution at a fixed time $t>0$. This issue is currently under investigation.

\section{Conclusions}

This paper compares solutions of the vortex blob and the Navier-Stokes equations, in the limit as both $\delta, \nu \rightarrow 0$. We show that while large scale features of the vortex sheet appear to converge [13], there are quantities such as the scaled oscillation frequency that differ in the limit of interest.

\section{Acknowledgements}

This work was partially supported by NSF Grant DMS0107187.

\section{References}

[1] Moore DW. The spontaneous appearance of a singularity in the shape of an evolving vortex sheet. Proc R Soc London A 1979;365:105-119.

[2] Krasny R. Computing vortex sheet motion. Proc Int Congr Math Kyoto 1990;1573-1583.

[3] Cowley SJ, Baker G, Tanveer S. On the formation of Moore curvature singularities in vortex sheets. J Fluid Mech 1999;378:233-267.

[4] Chorin AJ Bernard PS. Discretization of a vortex sheet, with an example of roll-up. J Comput Phys 1973;13:423429.

[5] Anderson C. A vortex method for flows with slight density variations. J Comput Phys 1985;61:417-444.

[6] Krasny R. Computation of vortex sheet roll-up in the Trefftz plane. J Fluid Mech 1987;184:123-155.

[7] Cottet G-H, Koumoutsakos PS. Vortex Methods: Theory and Practice. Cambridge: Cambridge University Press, 2000.

[8] Delort JM. Existence de nappes de tourbillon en dimension deux. J Am Math Soc 1991;4:553-586.

[9] Majda A. Remarks on weak solutions for vortex sheets 
with a distinguished sign. Indiana Univ Math J 1993;42(3): 921-939.

[10] Majda AJ, Bertozzi AL. Vorticity and Incompressible Flow. Cambridge: Cambridge University Press, 2002.

[11] Krasny R, Nitsche M. The onset of chaos in vortex sheet flow. J Fluid Mech 2002;454:47-69.

[12] Pullin DI. The large-scale structure of unsteady self-similar rolled-up vortex sheets. J Fluid Mech 1978;88:401-430.

[13] Tryggvason G, Dahm WJA, Sbeih K. Fine structure of vortex sheet roll-up by viscous and inviscid simulation. J Fluids Eng 1991;113:31-36.

[14] Nitsche M, Krasny R. A numerical study of vortex ring formation at the edge of a circular tube. J Fluid Mech 1994;276:139-161. 